\title{
An Efficient Face Tracker Using Active Shape Model
}

\author{
S. K. Murugaraja ${ }^{1}{ }^{2}$ K.Balamurali \\ ${ }^{1}$ Head Of The Department / Computer Science And Engineering Department,Anna University Chennai, \\ Gnanmani College Of Technology, Rasipuram, Namakkal -637214, India. \\ ${ }^{2}$ M.E Computer Science And Engineering Department, Anna University Chennai, Gnanmani College Of \\ Technology, Rasipuram, Namakkal -637214, India.
}

\begin{abstract}
To perform tracking in an illumination insensitive feature space, called the gradient logarithm field (GLF) feature space. The GLF feature mainly depends on the intrinsic characteristics of a face and is only marginally affected by the lighting source. The GLF feature is a global feature and does not depend on a specific face model, and thus is effective in tracking low-resolution faces. By using the GLF face tracking system, the modified image like editing image did not recognition. To overcome this problem, we propose, to find the original image of the editable image by using the outline dimension of the image. To recognition original face from the modified face, we track the inclusive (complete) region of the face. The Active Shape Model is used to recover the shape of the face and then find the face after comparing the faces.
\end{abstract}

\subsection{DOMAIN DESCRIPTION}

\section{Introduction}

\subsubsection{COMPUTER IMAGING}

Can be defined a acquisition and processing of visual information by computer. Computer representation of an image requires the equivalent of many thousands of words of data, so the massive amount of data required for image is a primary reason for the development of many sub areas with field of computer imaging, such as image compression and segmentation .Another important aspect of computer imaging involves the ultimate "receiver" of visual information in some case the human visual system and in some cases the human visual system and in others the computer itself. Computer imaging can be separate into two primary categories:

1. Computer Vision

2. Image Processing

(In computer vision application the processed images output for use by a computer, whereas in image processing applications the output images are for human consumption). These two categories are not totally separate and distinct. The boundaries that separate the two are fuzzy, but this definition allows us to explore the differences between the two and to explore the difference between the two and to understand how they fit together.

Historically, the field of image processing grew from electrical engineering as an extension of the signal processing branch, whereas are the computer science discipline was largely responsible for developments in computer vision.

\subsubsection{COMPUTER VISION}

Computer vision computer imaging where the application doses not involve a human being in visual loop. One of the major topics within this field of computer vision is image analysis.

> Image Analysis: involves the examination of the image data to facilitate solving vision problem. The image analysis process involves two other topics:

- Feature Extraction: is the process of acquiring higher level image information, such as shape or color information.

-Pattern Classification: is the act of taking this higher -level information and identifying objects within the image.

$>$ Computer vision systems are used in many and various types of environments, such as:
* Manufacturing Systems
* Medical Community
* Law Enforcement
* Infrared Imaging
* Satellites Orbiting

\subsubsection{IMAGE PROCESSING}

The major topics within the field of image processing include: 
- Image restoration

- Image enhancement

- Image compression

\section{IMAGE RESTORATION}

Is the process of taking an image with some known, or estimated degradation, and restoring it to its original appearance. Image restoration is often used in the field of photography or publishing where an image was somehow degraded but needs to be improved before it can be printed.

\section{IMAGE ENHANCEMENT}

Involves taking an image and improving it visually, typically by taking advantages of human Visual Systems responses. One of the simplest enhancement techniques is to simply stretch the contrast of an image. Enhancement methods tend to be problem specific. For example, a method that is used to enhance satellite images may not suitable for enhancing medical images.

Although enhancement and restoration are similar in aim, to make an image look better. They differ in how they approach the problem. Restoration method attempt to model the distortion to the image and reverse the degradation, where enhancement methods use knowledge of the human visual systems responses to improve an image visually.

\section{IMAGE COMPRESSION}

Involves reducing the typically massive amount of data needed to represent an image. This done by eliminating data that are visually unnecessary and by taking advantage of the redundancy that is inherent in most images. Image processing systems are used in many and various types of environments, such as:

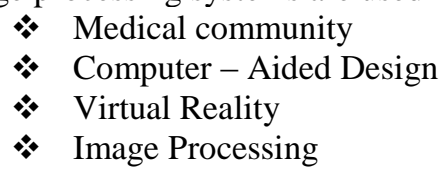

\subsection{PROJECT DESCRIPTION}

Object tracking is an important first step in many computer vision and pattern recognition applications. Research on object tracking has been undertaken for more than twenty years and has yielded many promising algorithms with encouraging results. In the last decade, a large number of surveillance cameras have been installed in cities all over the world. The videos captured by these networked cameras contain a lot of useful information, but the sheer volume of video data makes it very difficult to extract useful intelligence information within a reasonable timeframe. While camera designs are well-developed, the software supporting these applications lags behind. With increasing demands placed on video-based surveillance applications, low resolution face tracking under illumination changes is a challenging problem as the amount of information a tracker can use is limited.

One effective method to deal with variations in the appearance of a tracked object is to automatically update the tracking model using an online learning approach. For example, the online adaptive appearance model frequently updates the object template, while the online boosting model automatically adjusts the boosting classifier. These online trackers have been highly successful in handling small variations in pose and occlusion, but do not work well for tracking a face when major illumination changes occur. Tracking with a face model, such as an AAM or a physics-based face model, can be employed to handle significant face variations. Because the model-based approach requires that the image of the face be precisely aligned to a face model, this approach does not perform well in tracking low resolution faces. Another possible approach to this problem is to perform illumination normalization/compensation before tracking.

Existing illumination normalization/compensation algorithms can be roughly classified into two categories: model-based approaches and model-free approaches. Methods based on the 3D morphable model (3DMM) and the face reflectance model are typical examples of model-based approaches. Model-based methods require a high resolution face image to perform image analysis and to fit the model, and so may not be effective for tracking low resolution faces. Under a model-free approach, the underlying rationale is to find illumination invariant features. Model-free methods have been used principally for object recognition, and have not been extensively applied to resolve the low resolution face tracking problem.

To perform tracking in an illumination insensitive feature space, called the gradient logarithm field (GLF) feature space. The GLF feature mainly depends on the intrinsic characteristics of a face and is only marginally affected by the lighting source. The GLF feature is a global feature and does not depend on a specific face model, and thus is effective in tracking low-resolution faces. By using the GLF face tracking system, the modified image like editing image did not recognition. To overcome this problem, we propose, to find the 
original image of the editable image by using the outline dimension of the image. To recognition original face from the modified face, we track the inclusive(complete) region of the face. The Active Shape Model is used to recover the shape of the face and then find the face after comparing the faces.

\section{Literature Survey \\ 2.1 VISUAL TRACKING AND RECOGNITION USING APPEARANCE-ADAPTIVE MODELS IN PARTICLE FILTERS}

We present an approach that incorporates appearance-adaptive models in a particle filter to realize robust visual tracking and recognition algorithms. Tracking needs modeling inter-frame motion and appearance changes whereas recognition needs modeling appearance changes between frames and gallery images. In conventional tracking algorithms, the appearance model is either fixed or rapidly changing, and the motion model is simply a random walk with fixed noise variance. Also, the number of particles is typically fixed. All these factors make the visual tracker unstable. To stabilize the tracker, we propose the following modifications: an observation model arising from an adaptive appearance model, an adaptive velocity motion model with adaptive noise variance, and an adaptive number of particles. The adaptivevelocity model is derived using a first-order linear predictor based on the appearance difference between the incoming observation and the previous particle configuration. Occlusion analysis is implemented using robust statistics. Experimental results on tracking visual objects in long outdoor and indoor video sequences demonstrate the effectiveness and robustness of our tracking algorithm. We then perform simultaneous tracking and recognition by embedding them in a particle filter. For recognition purposes, we model the appearance changes between frames and gallery images by constructing the intra- and extra-personal spaces. Accurate recognition is achieved when confronted by pose and view variations.

\subsection{BEYOND SEMI-SUPERVISED TRACKING: TRACKING SHOULD BE AS SIMPLE AS DETECTION,BUT NOT SIMPLER THANRE COGNITION}

We present a multiple classifier system for model-free tracking. The tasks of detection (finding the object of interest), recognition (distinguishing similar objects in a scene), and tracking (retrieving the object to be tracked) are split into separate classifiers in the spirit of simplifying each classification task. The supervised and semi-supervised classifiers are carefully trained on-line in order to increase adaptivity while limiting accumulation of errors, i.e. drifting. In the experiments, we demonstrate real-time tracking on several challenging sequences, including multi-object tracking of faces, humans, and other objects. We outperform other on-line tracking methods especially in case of occlusions and presence of similar objects.

\subsection{FACE DESCRIPTION WITH LOCAL BINARY PATTERNS: APPLICATION TO FACE RECOGNITION}

This paper presents a novel and efficient facial image representation based on local binary pattern (LBP) texture features. The face image is divided into several regions from which the LBP feature distributions are extracted and concatenated into an enhanced feature vector to be used as a face descriptor. The performance of the proposed method is assessed in the face recognition problem under different challenges. Other applications and several extensions are also discussed.

\subsection{REAL-TIME TRACKING VIA ON-LINE BOOSTING}

Very recently tracking was approached using classification techniques such as support vector machines. The object to be tracked is discriminated by a classifier from the background. In a similar spirit we propose a novel on-line AdaBoost feature selection algorithm for tracking. The distinct advantage of our method is its capability of on-line training. This allows to adapt the classifier while tracking the object. Therefore appearance changes of the object (e.g. out of plane rotations, illumination changes) are handled quite naturally. Moreover, depending on the background the algorithm selects the most discriminating features for tracking resulting in stable tracking results. By using fast computable features (e.g. Haar-like wavelets, orientation histograms, local binary patterns) the algorithm runs in real-time. We demonstrate the performance of the algorithm on several (publically available) video sequences.

\subsection{VISUAL TRACKING WITH ONLINE MULTIPLE INSTANCE LEARNING}

In this paper, we address the problem of learning an adaptive appearance model for object tracking. In particular, a class of tracking techniques called "tracking by detection" have been shown to give promising results at realtime speeds. These methods train a discriminative classifier in an online manner to separate the object from the background. This classifier bootstraps itself by using the current tracker state to extract positive and negative examples from the current frame. Slight inaccuracies in the tracker can therefore lead to incorrectly labeled training examples, which degrades the classifier and can cause further drift. In this paper we show that 
using Multiple Instance Learning (MIL) instead of traditional supervised learning avoids these problems, and can therefore lead to a more robust tracker with fewer parameter tweaks. We present a novel online MIL algorithm for object tracking that achieves superior results with real-time performance.

\subsection{OBJECT TRACKING: A SURVEY}

The goal of this article is to review the state-of-the-art tracking methods, classify them into different categories, and identify new trends. Object tracking, in general, is a challenging problem. Difficulties in tracking objects can arise due to abrupt object motion, changing appearance patterns of both the object and the scene, nonrigid object structures, object-to-object and object-to-scene occlusions, and camera motion. Tracking is usually performed in the context of higher-level applications that require the location and/or shape of the object in every frame. Typically, assumptions are made to constrain the tracking problem in the context of a particular application. In this survey, we categorize the tracking methods on the basis of the object and motion representations used, provide detailed descriptions of representative methods in each category, and examine their pros and cons. Moreover, we discuss the important issues related to tracking including the use of appropriate image features, selection of motion models, and detection of objects.

\subsection{ROBUST VISUAL TRACKING AND VEHICLE CLASSIFICATION VIA SPARSE REPRESENTATION}

In this paper, we propose a robust visual tracking method by casting tracking as a sparse approximation problem in a particle filter framework. In this framework, occlusion, noise and other challenging issues are addressed seamlessly through a set of trivial templates. Specifically, to find the tracking target in a new frame, each target candidate is sparsely represented in the space spanned by target templates and trivial templates. The sparsity is achieved by solving an regularized least squares problem. Then the candidate with the smallest projection error is taken as the tracking target. After that, tracking is continued using a Bayesian state inference framework. Two strategies are used to further improve the tracking performance. First, target templates are dynamically updated to capture appearance changes. Second, nonnegativity constraints are enforced to filter out clutters which negatively resemble tracking targets. We test the proposed approach on numerous sequences involving different types of challenges including occlusion and variations in illumination, scale, and pose. The proposed approach demonstrates excellent performance in comparison with previously proposed trackers. We also extend the method for simultaneous tracking and recognition by introducing a static template set, which stores target images from different classes. The recognition result at each frame is propagated to produce the final result for the whole video. The approach is validated on a vehicle tracking and classification task using outdoor infrared video sequences.

\subsection{ROBUST ONLINE APPEARANCE MODELS FOR VISUAL TRACKING}

We propose a framework for learning robust, adaptive, appearance models to be used for motion-based tracking of natural objects. The approach involves a mixture of stable image structure, learned over long time courses, along with 2 -frame motion information and an outlier process. An online EM-algorithm is used to adapt the appearance model parameters over time. An implementation of this approach is developed for an appearance model based on the filter responses from a steerable pyramid. This model is used in a motion-based tracking algorithm to provide robustness in the face of image outliers, such as those caused by occlusions. It is also provides the ability to adapt to natural changes in appearance, such as those due to facial expressions or variations in 3D pose. We show experimental results on a variety of natural image sequences of people moving within cluttered environments.

\subsection{A PHYSICS-BASED ANALYSIS OF IMAGE APPEARANCE MODELS}

Linear and multilinear models (PCA, 3DMM, AAM/ASM, and multilinear tensors) of object shape/appearance have been very popular in computer vision. In this paper, we analyze the applicability of these heuristic models from the fundamental physical laws of object motion and image formation. We prove that under suitable conditions, the image appearance space can be closely approximated to be multilinear, with the illumination and texture subspaces being trilinearly combined with the direct sum of the motion and deformation subspaces. This result provides a physics-based understanding of many of the successes and limitations of the linear and multilinear approaches existing in the computer vision literature, and also identifies some of the conditions under which they are valid. It provides an analytical representation of the image space in terms of different physical factors that affect the image formation process. Numerical analysis of the accuracy of the physics-based models is performed, and tracking results on real data are presented. 


\subsection{POSE-ROBUST ALBEDO ESTIMATION FROM A SINGLE IMAGE}

We present a stochastic filtering approach to perform albedo estimation from a single non-frontal face image. Albedo estimation has far reaching applications in various computer vision tasks like illuminationinsensitive matching, shape recovery, etc. We extend the formulation proposed in that assumes face in known pose and present an algorithm that can perform albedo estimation from a single image even when pose information is inaccurate. 3D pose of the input face image is obtained as a byproduct of the algorithm. The proposed approach utilizes class-specific statistics of faces to iteratively improve albedo and pose estimates. Illustrations and experimental results are provided to show the effectiveness of the approach. We highlight the usefulness of the method for the task of matching faces across variations in pose and illumination. The facial pose estimates obtained are also compared against ground truth.

\subsection{EXISTING SYSTEM}

\section{System Design}

In this existing to perform tracking in an illumination insensitive feature space, called the gradient logarithm field(GLF) feature space. The GLF based face tracking system, find the genuine (original) face from the low resolution image. The GLF, track the face by describing a small region of the face. The GLF feature is only effective for tracking low resolution faces not to track an original face of an adapted (modified) face.

\subsection{PROBLEM DEFINITION}

The model-based approach requires that the image of the face be precisely aligned to a face model, this approach does not perform well in tracking low resolution faces.

Model-free methods have been used principally for object recognition, and have not been extensively applied to resolve the low resolution face tracking problem.

\subsection{PROPOSED SYSTEM}

The proposed system, we find the original face of the custom-made (modified) image. For example, the original image was modified by changing the eyes etc., to track the authentic (true) face by comparing the outline dimension of both original and modified face. The proposed system, not only describe the small region of the face but also, it describe a complete region of the face. So, it is easy to track the original face.

\section{Systemarchitecture}

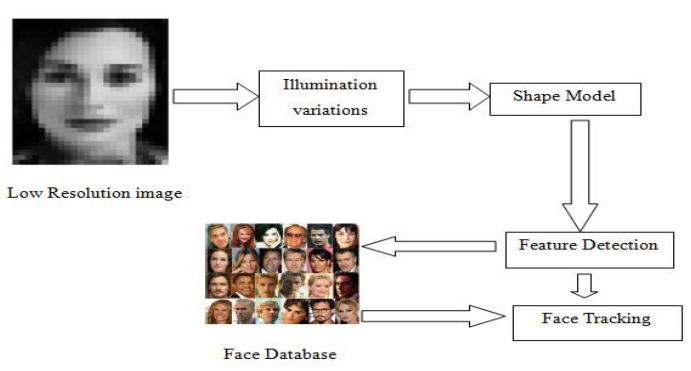

FIGURE 3.3.A SYSTEMARCHITECTURE

\section{DATA FLOW DIAGRA}

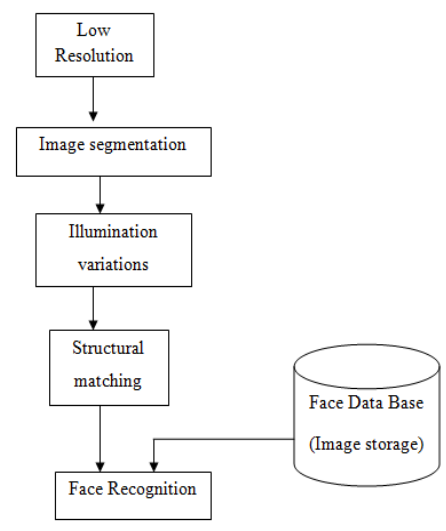

FIGURE 3.3.B DATA FLOW DIAGRAM 


\subsection{MODULES}

\section{System Models}

\subsubsection{IMAGE SEGMENTATION}

Segmentation is the process of partitioning a low resolution image into semantically interpretable regions. An image segmentation is the partition of an image into a set of nonoverlapping regions whose union is the entire image. The purpose of segmentation is to decompose the image into parts that are meaningful with respect to a particular application. A segmentation could be used for object recognition, occlusion boundary estimation within motion

\subsubsection{ILLUMINATION VARIATIONS}

A straightforward method of handling illumination variations is to separate the lighting source from image and extract the intrinsic features to characterize the face. However, it is an ill-posed open problem that may need additional models for the face, which is not applicable to low-resolution image. We show that under some reasonable assumptions, the performance degradations caused by lighting source changes can be alleviated.

\subsubsection{SHAPE RECOVERY}

Shape is another intrinsic property of an object which is invariant to changes in pose and illumination conditions. The importance of estimating the shape of an object has probably been the guiding force behind the vast amount of work that has been done to recover shape from images. The goal of SFS (Shape from Shading) research is to recover the image shape of an object from a single image. We propose an algorithm that transforms the general SFS to one of estimating the shape of an object with unit illuminant variation that can be addressed using a standard SFS approach.

\subsubsection{STRUCTURAL MATCHING}

ASM(Active Shape Model) is used for Structural matching. More robust under shape or image intensity variation. Statistical model representing the shape of faces: Point distribution model with $\mathrm{N}$ points Local appearance model for each point based on image gradient, Point and local appearance distributions learned by applying PCA(Principal Component Analysis) to a set of annotated images, The face can be expressed as the sum of a mean shape and a linear combination of basis shapes. Iterative fitting to find the points that best match the local appearance distributions under constraints imposed by shape.

\subsubsection{TRACKING WITH OTHER FACE VARIATIONS}

Face variations other than changes in illumination (such as changes in pose, expression and occlusion) will also change the appearance of the face being tracked which may cause the tracker to fail. The adaptive learning model in the particle filter framework can help to address such face variations. Finally, we track the original image by comparing the illumination variation images with other faces that are stored in image storage. The Structural matching face are also compare with other faces.

\section{Conclusion}

In this paper, we address the problem of tracking a low resolution face subject to illumination changes and shape model. This paper possesses a desirable property is it does not depend on a specific face model. Advantages of the active shape model techniques are their simplicity and applicability for use in conjunction with other methods. Another estimation framework is developed to reduce the general problem of recovering shape of an object with varying unknown illuminant variation to the one that can be addressed by the traditional SFS approaches.

\section{References}

[1] S. Stalder, H. Grabner, and L. Van Gool, "Beyond semi-supervised tracking: Tracking should be as simple as detection, but not simpler than recognition," in 2009.

[2] T. Ahonen, A. Hadid, and M. Pietikainen, "Face description with local binary patterns: Application to face recognition," in Dec.2006.

[3] H. Grabner, M. Grabner, and H. Bischof, "Real-time tracking via on-line boosting," on 2006

[4] S. Zhou, R. Chellappa, and B. Moghaddam, "Visual tracking and recognition using appearance-adaptive models in particle filters", in 2008 .

[5] B. Babenko, M. Yang, and S. Belongie, "Visual tracking with online multiple instance learning," in 2011.

[6] Yilmaz, O. Javed, and M. Shah, "Object tracking: A survey," ACM Comput. Surv., vol. 38, no. 4, p. 13, 2006.

[7] Jepson, D. Fleet, and T. El-Maraghi, "Robust online appearance models for visual tracking," IEEE Trans. Pattern Anal. Mach. Intell., vol. 25, no. 10, pp. 1296-1311, Oct. 2003.

[8] X. Mei and H. Ling, "Robust visual tracking and vehicle classification via sparse representation," IEEE Trans. Pattern Anal. Mach. Intell., vol. 33, no. 11, pp. 2259-2272, Nov. 2011.

[9] Y. Xu and A. Roy-Chowdhury, "A physics-based analysis of image appearance models," IEEE Trans. Pattern Anal. Mach. Intell., vol. 33, no. 8, pp. 1681-1688, Aug. 2011.

[10] S. Biswas and R. Chellappa, "Pose-robust albedo estimation from a single image," in Proc. Conf. Comput. Vis. Pattern Recognit., 2010. 